\title{
Use of latex agglutination technique for detecting Legionella pneumophila (serogroup 1) antibodies
}

\author{
M G Holliday
}

\begin{abstract}
Following the outbreak of Legionnaires' disease in Stafford in 1985, 500 serum samples were submitted to the indirect immunofluorescence antibody test and a latex agglutination. Latex agglutination using ultrasonically disrupted Legionella pneumophila antigens coupled to latex particles, proved a rapid, simple method for detecting circulating antibodies to $L$ pneumophila in a one minute slide latex agglutination test. There was good correlation with the indirect immunofluorescence antibody test (IFAT), and the specificity and sensitivity with respect to a diagnostic result were $98.3 \%$ and $97.6 \%$, respectively, using a series of well characterised sera. The latex agglutination test seems well suited as a screening test for presumptive cases of Legionnaires' disease; the latex reagent is easy to prepare and seems to remain stable at $4^{\circ} \mathrm{C}$ for up to six months.
\end{abstract}

In United Kingdom laboratories serodiagnosis of Legionnaires' disease is usually made using either the indirect immunofluorescence antibody test (IFAT) ${ }^{1}$ or the rapid microagglutination test (RMAT). ${ }^{2}$ The sensitivity and specificity of these tests have been fully investigated using large series of well documented sera and have been shown to be about $80^{\circ} \%$ and $100 \%$, respectively, with a concordance between the two tests of $96.5 \%{ }^{3}{ }^{3}$

Counterimmunoelectrophoresis (CIE) and double immunodiffusion (DID) have also been evaluated using a large number of sera, but they failed to show good correlation with IFAT, the concordance being only $69.8 \%$ for CIE and $39.8^{\circ}$ o for DID. Neither test is recommended for routine use. ${ }^{4}$

A simple latex agglutination test has been described which seems to be a useful screening test for presumptive cases of Legionnaires' disease, ${ }^{5}$ but relatively few sera were examined.

This study reports the results obtained by examining a large series of well characterised sera by Legionnaires' disease to assess better sensitivity, specificity, and positive and negative predictive values.

\section{Methods}

Five hundred sera obtained from patients between April and September 1985 were included in this investigation. All sera were sent to the laboratory for Legionella serology following the outbreak in 1985 of Legionnaires' disease in Stafford and had IFAT tests performed on them at the Public Health Laboratories in Manchester, Preston, or Birmingham.

All sera were stored, coded, and randomised, as described previously. ${ }^{5}$ The criteria for a definite diagnosis of Legionnaires' disease in hospital inpatients were clinical evidence of pneumonia or chest infection, supported by serological evidence of infection with a four-fold rise in IFAT titres to at least $1 / 64$, or a reproducible titre of $1 / 128$ on a single specimen and prior exposure at the Stafford District General Hospital (SDGH). For outpatients the criteria were clinical evidence of chest infection supported by a serum IFAT titre of $1 / 64$ and associated exposure at the SDGH. ${ }^{4}$

Serum samples from patients with confirmed Legionnaires' disease and with IFAT titres of $>1 / 64$ are classed as having diagnostic IFAT titres.

Sixty patients from whom 162 sera were tested had a definite diagnosis of Legionnaires' disease (48 inpatients and 12 outpatients). A further 23 patients from whom 36 sera with positive IFAT titres were tested did not fulfil the full diagnostic criteria. Sixteen sera from 14 members of staff who had positive IFAT titres were tested, as were 286 sera with IFAT titres of $<1 / 16$ from 286 other hospital patients who were not considered to have had Legionnaires' disease.

Rabbit antisera to Legionella pneumophila serogroups 1 to 8 were kindly provided by Dr A G Taylor, Division of Microbial Reagents and Quality Control (DMRQC), Colindale, and a rabbit antiserum to ultrasonicated $L$ pneumophila serogroup 1 cells was generously supplied by Dr J Gray and Mr A Barrett, PHLS, Stoke on Trent.

LATEX ANTIGEN REAGENT

The antigen was made from ultrasonically disrupted $L$ pneumophila serogroup 1 cells $^{6}$ (Pontiac strain NCTC 11191) harvested from five day growth on BCYE plates (Oxoid Ltd, Basingstoke, England).

The latex sensitisation was by a modification of the method of Severin as follows. ${ }^{7}$ One volume of optimally diluted antigen was mixed with one volume of well mixed latex particles $(0.81 \mu \mathrm{m}$. Difco Laboratories, Surrey, England), shaken well, and incubated at $37^{\circ} \mathrm{C}$ for two hours. Subsequently, two volumes of glycine buffered saline plus $0.1 \%$ bovine serum albumin (GBS-BSA) were 
added. This was incubated at room temperature with occasional shaking for one hour before being centrifuged. The supernatant was discarded and the latex particles were resuspended in four volumes of GBS-BSA. This reagent can be stored at $4^{\circ} \mathrm{C}$ for at least six months.

The optimal dilution of antigen for sensitisation must be determined by sensitising small aliquots of latex with different dilutions of antigen as above and testing against dilutions of known positive sera. The antigen dilution giving maximum clarity of agglutination and highest antibody titres should be used.

\section{LATEXT AGGLUTINATION TEST}

This was performed by mixing $20 \mu$ l of latex reagent and $20 \mu \mathrm{l}$ of serum on a black glass slide for exactly one minute before observing. Visible agglutination was regarded as positive and no agglutination was considered to be negative. Trace agglutination was ignored. Sera were titrated by testing serial dilutions made in GBS-BSA as above. The highest dilution showing visible agglutination was taken as the end point.

\section{DEFINITIONS}

The specificity, sensitivity, and negative and positive predictive values for latex agglutination were determined, ${ }^{8}$ and the following definitions applied. True positive were positive results from patients with confirmed Legionnaires' disease and IFAT titres of more than or equal to $1 \cdot 16$. False positive were positive results from patients with no evidence of Legionnaires' disease. True negative were negative results from patients with no evidence of Legionnaires' disease. False negative were negative results from patients with Legionnaires' disease and IFAT titres of more than or equal to $1 \cdot 16$.

\section{Results}

The 60 confirmed cases of Legionnaires' disease produced 134 IFAT positive sera. A diagnostic IFAT titre was given by 82 of these, of which 80 were latex agglutination positive. Fifty two sera gave positive, but not diagnostic, IFAT titres, and 48 of these were latex agglutination positive, while 28 sera

Table 1 Comparison of Legionella IFAT and LA results in different patient groups

\begin{tabular}{|c|c|c|}
\hline Patient group & Total & $\begin{array}{l}\text { Number (\%) of sera } \\
\text { giving a positive latex } \\
\text { agglutination result }\end{array}$ \\
\hline \multicolumn{3}{|l|}{ Confirmed Legionnaire's disease: } \\
\hline $\begin{array}{l}\text { Sera with diagnostic IFAT titres } \\
\text { Sera with non-diagnostic IFAT titres } \\
\text { Sera with IFAT titres of }<1 / 16\end{array}$ & $\begin{array}{l}82 \\
52 \\
28\end{array}$ & $\begin{array}{l}80(97 \cdot 6) \\
48(92 \cdot 3) \\
0\end{array}$ \\
\hline $\begin{array}{l}\text { Patients not fulfilling full diagnostic criteria } \\
\text { with non-diagnostic IFAT titres }\end{array}$ & 36 & $32(88 \cdot 8)$ \\
\hline \multicolumn{3}{|l|}{ Staff } \\
\hline $\begin{array}{l}\text { Sera with diagnostic IFAT titres } \\
\text { Sera with non-diagnostic IFAT titres }\end{array}$ & $\begin{array}{l}7 \\
9\end{array}$ & $\begin{array}{l}7(100) \\
6(66 \cdot 6)\end{array}$ \\
\hline \multicolumn{3}{|c|}{ Patients with no evidence of Legionnaire's disease } \\
\hline Sera with IFAT titres of $<1 / 16$ & 286 & $5(1 \cdot 7)$ \\
\hline \multicolumn{3}{|l|}{ Rabbit anti-L pneumophila sera: } \\
\hline $\begin{array}{l}\text { Serogroup } 1 \text { antisera } \\
\text { Serogroups } 2-8 \text { antisera }\end{array}$ & $\stackrel{2}{\text { One of each }}$ & $2(100)$ \\
\hline
\end{tabular}

Table 2 Comparison of latex agglutination test results with IFAT titres in 186 IFAT positive sera

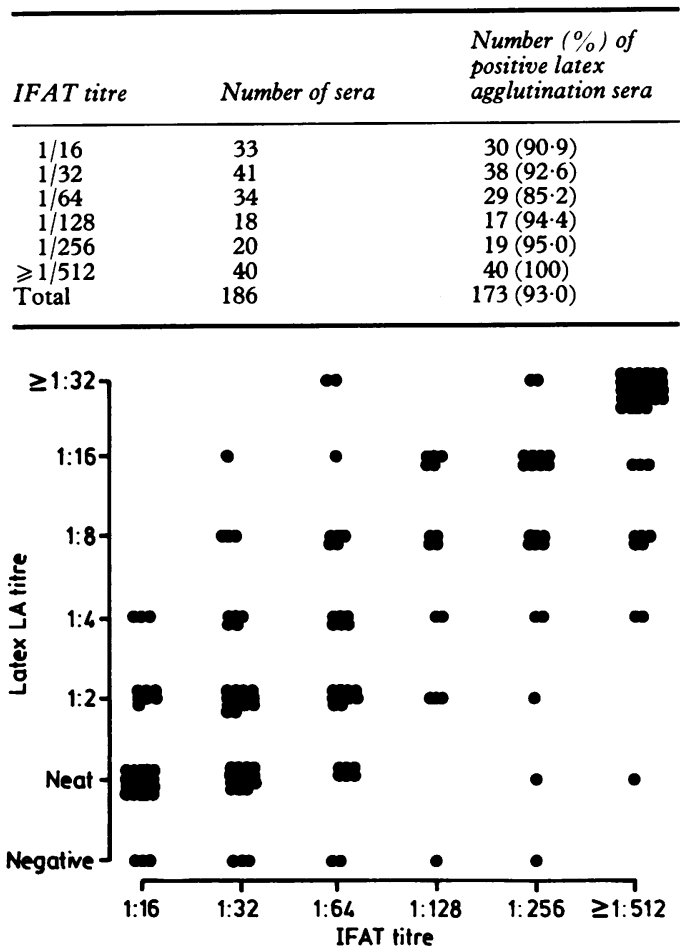

Figure Comparison of IFAT and latex agglutination titres in 186 sera with IF $A$ T titres of $\geqslant 1: 16$.

were negative by both IFAT and latex agglutination (table 1 ).

The patients not fulfilling the full diagnostic criteria produced 36 sera that were IFAT positive but lacked diagnostic titres, and of these 32 were latex agglutination positive.

Seven sera from members of staff giving diagnostic IFAT titres were all latex agglutination positive, and of nine sera giving nondiagnostic IFAT titres, six were latex agglutination positive. Of the remaining 286 sera with IFAT titres of less than $1 / 16$, five were latex agglutination positive.

Two rabbit antisera to $L$ pneumophila serogroup 1 were latex agglutination positive, rabbit antisera to $L$ pneumophila serogroups 2 to 8 were all latex agglutination negative. These antisera were specific for their given serogroup and did not cross-react with serogroup 1 when tested by IFAT.

The relation between IFAT titres and latex agglutination results in 186 IFAT positive sera is shown in table 2 , while that between IFAT titres and latex agglutination titres is shown in the figure.

When latex agglutination screening tests were done in triplicate, no discrepancies were found in results, and when sera were subsequently titrated by latex agglutination in triplicate, only occasional differences in titre were observed, these being of the order of one to two-fold dilutions higher or lower only.

\section{Discussion}

Table 1 shows that the latex reagent seems to be specific for $L$ pneumophila serogroup 1 , although this cannot be established with certainty as only a limited number of rabbit antisera were used. A larger number of human 
sera with antibodies to different $L$ pneumophila serogroups must be tested to confirm this.

The correlation shown between latex agglutination and IFAT, with sera showing diagnostic IFAT titres was excellent, both with conformed cases of Legionnaires' disease and staff $(97.5 \%$ and $100 \%$ concordance, respectively), but the correlation seen with confirmed cases of Legionnaires' disease with non-diagnostic IFAT titres was only slightly less at $92.3 \%$. These figures may be biased due to the fact that the sera were all from one epidemic and the causative strain of $L$ pneumophila was the same as that used to make the latex agglutination antigen (Pontiac). Perhaps if a more heterogeneous group of positive sera from cases caused by other strains had been tested these figures may have differed.

It has been suggested that "the most important test variable in the case of an illness of low prevalence, such as Legionnaires' disease, is the specificity". ${ }^{3}$ The specificity of IFAT for a diagnostic result is $100 \%$ and, compared with this, latex agglutination has a specificity of $98.3 \%$ using the definitions made earlier.

It is difficult to calculate the true sensitivity of latex agglutination because the IFAT result was used to confirm the diagnosis of Legionnaires' disease for the patients in the study. This gives the IFAT an apparent sensitivity of $100 \%$. Compared with this, and using the definitions made earlier, latex agglutination has an apparent sensitivity of $97.6 \%$. The sensitivity of IFAT with respect to a diagnostic result, however, is not $100 \%$ but $79 \%$, with a further $10 \%$ of specimens giving a positive, but not diagnostic result. ${ }^{3}$ The true sensitivity of latex agglutination is probably somewhat less than $79 \%$.

Assuming the prevalence of Legionnaires' disease to be between $1 \%$ and $5 \%{ }^{3}$ the predictive value of a positive latex agglutination result was calculated to be between $36.7 \%$ and $75.1 \%$, and the predictive value of a negative latex agglutination result was calculated to be between $99.97 \%$ and $99.87 \%$. These figures do not, however, take into account the IFAT positive sera from patients not fulfilling the full diagnostic criteria, or from members of staff, as it was not possible to classify these groups accurately. It is likely, given the circumstances, that many of these subjects had been in contact with legionellae and were producing a specific antibody response to them, but insufficient information and follow up sera were available to permit inclusion as genuine outbreak cases. ${ }^{4}$ It has been suggested that positive but non-diagnostic IFAT titres may be good indicators of Legionnaires' disease ${ }^{3}$ and the results from this outbreak would tend to support this theory. It is worthy of note that, of the 36 sera from patients and nine from staff in these groups, $88.8 \%$ and $66.6 \%$, respectively, were latex agglutination positive, indicating that latex agglutination may be positive in more than two thirds of sera with "suggestive" IFAT titres.
When the latex agglutination result was compared with that of the IFAT titre (table 2) latex agglutination was positive in sera with IFAT titres of more than $1 / 128$ in slightly more cases than in sera with IFAT titres of less than $1 / 128$. Sera with IFAT titres of $1 / 64$ gave proportionally the fewest latex agglutination positive reactions, but the reason for this is unknown.

When the relation between LA and IFAT titres was examined (figure), a roughly linear correlation was seen with most sera, but there were several sera that showed discrepancies (notably sera with varying IFAT titres that were latex agglutination negative), and it is suggested that in some cases latex agglutination titres may be misleading and are therefore unnecessary.

Seven patients with Legionnaires' disease provided paired sera which showed a fourfold or greater rise in titre by IFAT. In each case the latex agglutination titre also showed a four-fold or greater rise.

The latex agglutination test probably detects predominantly IgM, in common with other agglutination tests for legionella, while IFAT detects both IgG and IgM, ${ }^{2}$ and in sera containing predominantly IgG IFAT may be positive while latex agglutination is negative. This could account for the discrepancies observed in this study.

The latex agglutination test is an extremely simple and inexpensive test which is well within the capability of most laboratories. It requires no expensive or sophisticated equipment and no specific expertise. The rapidity of the test, coupled with its high specificity and sensitivity, and correlation with IFAT results make the latex agglutination test a candidate for a useful screening test for presumptive cases of Legionnaires' disease. Positive sera can be forwarded to reference laboratories for confirmation by standard methods such as IFAT. The latex reagent is easy to prepare and seems to remain stable at $4^{\circ} \mathrm{C}$ for up to six months.

I thank the staff of the Public Health Laboratories at Preston Manchester, and Birmingham who performed all the IFAT testing, and the staff of the Microbiology Department Stafford, especially Mr I L McCartney, without whom none of this work would have been possible.

1 Harrison TG, Taylor AG. Diagnosis of Legionella pneumophila infections by means of formolised yolk sac antigens. $J$ Clin Pathol 1982;35:211-4.

2 Harrison TG, Taylor AG. A rapid microagglutination tes for the diagnosis of Legionella pneumophila (serogroup 1 ) infection. J Clin Pathol 1982;35:1028-31.

3 Harrison TG, Dournon E, Taylor AG. Evaluation of sensitivity of two serological tests for diagnosin pneumonia caused by Legionella pneumophila serogroup 1. J Clin Pathol 1987;40:77-82.

4 Holliday MG. Counterimmunoelectrophoresis and double immunodiffusion techniques in the detection of $\mathrm{Legion}-$ illa ella pneur

5 Holliday MG. Latex agglutination test for Legionella antibody. Lancet 1986;ii:465-6.

6 Holliday MG. The diagnosis of Legionnaires' Disease by counterimmunoelectrophoresis. J Clin Pathol 1980;33: counterim

7 Severin WPJ. Latex agglutination in the diagnosis of meningococcal meningitis. J Clin Pathol 1972;25:1079-82.

8 Galen RS, Gambino SR. Beyond normality. The predictive value and efficiency of medical diagnosis. New York: Wiley John \& Sons: 1975. 